\title{
The Power Spectra of Good Codes for Partial Response Channels
}

\author{
Xiao Ma, Aleksandar Kavčić and Michael Mitzenmacher \\ Division of Engineering and Applied Sciences, Harvard University \\ Cambridge, MA 02138, USA \\ e-mail: kavcic@eecs.harvard.edu
}

\section{EXtended Summary}

Recently, a serial concatenation coding scheme was proposed to approach the capacity of a partial response (PR) channel [1]. The design of the inner trellis code (named a matched information rate code (MIRC)) relies on the firstorder statistics (marginal distribution) of the (sub)optimal discrete input process. In this paper, we investigate the second-order statistics of the MIRC sequence.

Consider a linear Gaussian channel [2]. The wellknown water-filling theorem specifies a Gaussian process that achieves the capacity under the (input) power constraint. If the channel has spectral nulls, the power spectral density (p.s.d.) of the optimal Gaussian input process must vanish at those spectral nulls. Therefore, if the input variables are not constrained to finite constellations, good codes (in the sense defined in [3]) should match the spectral nulls. However, this may not be the case if input variables are limited to a finite constellation. In this paper, we will compute the power spectral density (p.s.d.) of the suboptimal input process under the constraint that the input at time $t$ can only take values from $\{ \pm A\}$. In this case, the channel is also known as a partial response ( $\mathrm{PR}$ ) channel, which is a basic model for digital recording systems [4]. In order to compute the p.s.d. of the discrete input process, a direct numerical method is utilized and justified. In contrast to analytical methods presented, e.g. in [5], the numerical method applies to most hidden Markov chains of interest. The outline of this work is listed as follows.

1. Introduce trellises of several types by generalizing the trellis representation of the PR channels [6]. Then define the input process by assigning a nonzero transition probability to each branch of a given trellis.

2. Find the (near) optimal (in the sense that the mutual information rate is maximized) transition probabilities by applying Kavčić's algorithm [7] to a fixed trellis.

3. Define a hidden Markov process $\underline{X}$ by treating branches of the trellis as vertexes of a new graph with edges properly defined. Then compute the autocorrelation coefficients $R_{x}(k)=\mathbf{E}\left(X_{1} X_{1+k}\right)$ for $-N \leq k \leq N$ and the partial sum $S_{x}^{(N)}(f)=\sum_{-N \leq k \leq N} \exp (-2 \pi f k \sqrt{-1})$.

The p.s.d. $S_{x}(f)$ of $\underline{X}$ is well approximated by $S_{x}^{(N)}(f)$ for suitably large $N$ because $S_{x}^{(N)}(f)$ is geometrically and uniformly convergent to $S_{x}(f)$. This is true because

Theorem: If the finite Markov model is irreducible and aperiodic, then there exist constants $c>0$ and $0<r<1$ such that $\left|R_{x}(k)\right| \leq c r^{k}$.

Proof: The proof, which is omitted here, is based on the convergence of the given Markov chain. More properties related to the convergence of a general Markov chain can be found, for example, in [8].

\footnotetext{
${ }^{1}$ This work was supported by the National Science Foundation under Grant No. CCR-0118701.
}

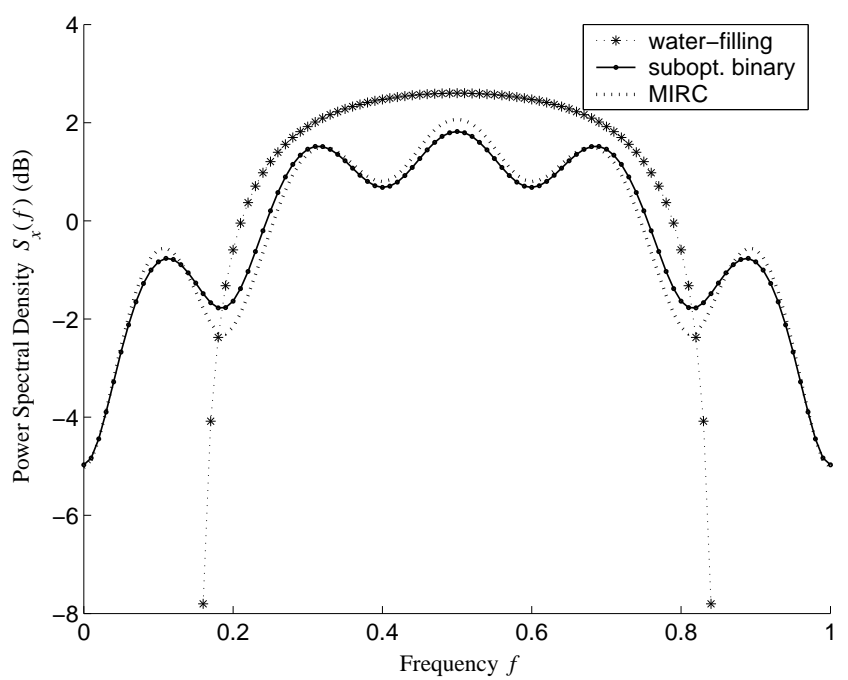

Fig. 1: The power spectral densities of the optimal continuous input process (specified by the water-filling theorem), a suboptimal binary input process (obtained by Kavčić's algorithm), and the constructed MIRC in [1] based on this binary process. The channel considered here is the dicode channel and $\mathrm{SNR}=0 \mathrm{~dB}$.

Numerical results (see Fig. 1) illustrate that (i) the main powers of the suboptimal binary input process concentrate in the frequency band where the water-filling solution does not vanish and (ii) the MIRC sequence [1] matches the suboptimal process very well in terms of second-order statistics and does have low powers (but not zero) at channel spectral nulls.

\section{REFERENCES}

[1] A. Kavčić, X. Ma, and N. Varnica, "Matched information rate codes for partial response channels." submitted to IEEE Trans. Inform. Theory. It was presented in part at ISIT'2002, Lausanne, Switzerland, June 30-July 5, 2002., June 2002.

[2] G. D. Forney, Jr. and G. Ungerboeck, "Modulation and coding for linear Gaussian channels," IEEE Trans. Inform. Theory, vol. 44, pp. 2384-2415, October 1998.

[3] S. Shamai and S. Verdú, "The empirical distribution of good codes," IEEE Trans. Inform. Theory, vol. 43, pp. 836-846, May 1997.

[4] K. A. S. Immink, P. H. Siegel, and J. K. Wolf, "Codes for digital recorders," IEEE Trans. Inform. Theory, vol. 44, pp. 22602299, October 1998.

[5] A. Gallopoulos, C. Heegard, and P. H. Siegel, "The power spectrum of run-length-limited codes," IEEE Trans. Commun., vol. 37, pp. 906-917, Sept. 1989.

[6] G. D. Forney Jr., "The Viterbi algorithm," Proc. IEEE, vol. 61, pp. 268-278, March 1973.

[7] A. Kavčić, "On the capacity of Markov sources over noisy channels," in Proceedings IEEE Global Communications Conference 2001, (San Antonio, Texas), November 2001.

[8] J. S. Rosenthal, "Convergence rates for Markov chains," SIAM Review, vol. 37, no. 3, pp. 387-405, 1995. 